\title{
Apports en DHA (acide gras oméga-3) par les poissons et les fruits de mer consommés en France
}

Jean-Marie BOURRE ${ }^{1}$

Philippe PAQUOTTE ${ }^{2}$

1 INSERM, U705; CNRS, UMR 7157, 200 rue du Faubourg Saint Denis.

75745 Paris cedex 10

<jean-marie.bourre@fwidal.inserm.fr> ${ }^{2}$ OFIMER (Office National Interprofessionnel des Produits de la Mer et de l'Aquaculture), 76/78 rue de Reuilly, 75012 Paris, France
Article reçu le 20 juin 2006 accepté le 20 juillet 2006

\begin{abstract}
This present work measures the contributions of seafood (finfish and shellfish, wild and farmed) to the intakes of DHA in France, year 2005, and consequently to the French recommended daily intakes (RDA) of DHA. For this purpose, we measured the concentrations of DHA in each individual seafood by analysis of many published data. We also determined the exact consumption of the main products of seafood (fishing and farming) in France (year 2005) using the modified method of the dietary intake measurement defined by the FAO.

For year 2005, the 34 species of finfish and shellfish whose DHA contents are known account for $150 \%$ of the RDA of this omega-3 fatty acid. Taking all the species individually and extrapolating to include those whose DHA contents are not known, gives $156 \%$ of the RDA. The 10 greatest contributors to the DHA intake in the present diet are: salmon (47.6 mg/day/person), sardine (28.4), tuna (20.5, mackerel (15.7), herring (12.4), Alaskan pollock (8.9), cod (6.9), trout (6.5), hake (4.7), saithe (4.6). The 5 species of oily fish (salmon, sardine, tuna, mackerel and herring) provide $63 \%$ of the DHA intake, and thus approx $95 \%$ of the French RDA.

Besides DHA, seafood contains low amounts of ALA, but it can very high in farmed fish fed on rapeseed or linseed products.

Omega-3 fatty acids (including EPA and DHA) have an important role in human diet, both for prevention and the therapy of different pathologies. Data from many epidemiological studies has shown an inverse association between fish consumption, generally oily fish, and reductions in certain diseases. Although number of people do not lack dietary DHA, others, mainly pregnant women and nursing mothers and those people whose life styles or socio-economic positions restrict their seafood intakes, would benefit greatly from an increased intake of this omega-3 fatty acid.
\end{abstract}

Key words: fish, shellfish, seafood, fishing, aquaculture, DHA, omega-3 polyunsaturated fatty acids, lipids

\section{Introduction}

Les études épidémiologiques, comme celles d'observation, montrent nombre de déséquilibres alimentaires, sources de déficits importants dans la consommation de certains nutriments, induisent des problèmes de santé publique. Parmi ces nutriments, les acides gras oméga-3 sont impliqués. Toutefois, une discussion importante concerne les réels apports alimentaires en acides gras polyinsaturés oméga-3. En effet, le déficit en ALA (acide alpha-linoléique, de la famille (n-3)) alimentaire ne fait pas de doute en France [1-3], comme dans de multiples autres pays. Les populations n'absorbent dans leur alimentation que la moitié seulement (voire moins) de l'ALA indiqué dans les ANC [4]. Cette insuffisance quantitative ne permet vraisemblablement pas $d^{\prime}$ 'assurer les besoins en DHA, qui lui est dérivé ; d'autant que le rendement de conversion est très faible. II convient donc de puiser ce DHA dans d'autres aliments, les poissons et fruits de mer au premier chef. Or, I'apport alimentaire en DHA (l'autre acide gras oméga-3 qui bénéficie d'ANC en France, avec l'ALA) est sujet à débat. D'autant que l'apport en acides gras oméga-3, ALA et/ou $D H A$, peut être augmenté en nourrissant les animaux de manière pertinente [5-7]. En effet, les teneurs en ALA (et, dans une moindre mesure du DHA) dans les produits issus d'animaux sont considérablement accrues par I'utilisation de graines de lin en alimentation animale, [2, 8]. De ce fait, les viandes, notamment celle de porc [9], la volaille et surtout les œufs [10], peuvent contribuer notablement à la couverture en acides gras oméga-3.

II n'en reste pas moins vrai que la source majeure de DHA alimentaire est constituée par les poissons et les fruits de mer, et ce d'autant qu'ils sont plus gras. Leur offre se compose de beaucoup d'espèces [11], qui, associées à un large éventail de préparations culinaires, permet de les recommander à tout âge, dans toutes les conditions de vie, de lieux, et pour tout type de repas. La perception de leur qualité dépend dans une certaine mesure du contenu lipidique [12,13]. La part des poissons dans la consommation des Français a été étudiée [14], mais pas dans le détail de chaque espèce, ni en incluant les fruits de mer, et sans préciser les apports consécutifs en DHA.

L'objectif de ce travail est donc de mesurer, en France, la consommation exacte des principaux poissons et fruits de mer (les produits animaux aquatiques, c'est-à-dire les produits de la pêche et 
de l'aquaculture), de calculer leurs teneurs en DHA, par analyse des données publiées, et de déterminer ainsi la contribution des poissons et fruits de mer à la couverture en ces nutriments, évaluée en \% des ANC (apports nutritionnels conseillés).

De nombreuses données, issues de multiples études épidémiologiques, ont mis en parallèle la consommation de poissons, généralement gras, avec la réduction de diverses pathologies. Des essais de prévention ou même thérapeutiques, en intervention, ont ensuite été réalisés. Ils ont reposé sur une prescription soit de consommation de poisson, soit de capsules d'huile de chair de poisson. Or, la principale caractéristique de ces poissons gras est d'être riches en acides gras oméga-3 $[15,16]$, sachant qu'ils apportent d'autres nutriments.

La consultation des banques de données montre qu'il existe plusieurs centaines de publications, portant sur les modèles animaux et l'homme, montrant les effets des acides gras oméga-3, en particulier ceux des huiles de poissons, dans le cadre de la prévention des maladies cardiovasculaires ischémiques, et de l'infarctus cérébral (attaque) [17-19]. Les plus hautes instances mondiales, notamment du domaine cardiologique recommandent 2 parts de poisson par semaine (dont au moins une de poisson gras). Par exemple, il a été calculé que, chez les faibles consommateurs de poissons et fruits de mer, tout accroissement de consommation alimentaire de poisson de $20 \mathrm{~g} / j$ jour diminue de $7 \%$ le risque de mortalité cardiovasculaire [20]. Sachant que les pertes lors de certaines préparations ne sont pas négligeables, et que l'enrichissement en acides gras indésirables peut être notable, il est intéressant de noter que la consommation de poisson frit (de thon en l'occurrence, dans cette étude) induit une augmentation du risque cardio-vasculaire, ce que ne fait pas la consommation de thon cuit autrement [21].

Par ailleurs, la consommation de poisson est en relation avec une réduction du risque de certains cancers, notamment du sein [22] et du colon [23, 24]. Les acides gras oméga-3 ont aussi pour propriété (parmi d'autres) de lutter contre l'inflammation. Par conséquent, outre les maladies cardiovasculaires ischémiques, d'autres pathologies font l'objet d'investigations: notamment rhumatologiques ou dermatologiques (le psoriasis), et même les maladies inflammatoires de l'intestin [25].

Dans un autre domaine, les acides gras oméga-3 sont parmi les nutriments essentiels qui interviennent sur les structures et les fonctions du cerveau, y compris cognitives [26, 27]. Ils agissent à tous les niveaux, notamment sur la neurotransmission cérébrale [28]. Récemment, il a été montré une relation entre la consommation de poissons et la prévention de certaines maladies psychiatriques, dont la dépression, la maladie bipolaire et la démence, ainsi que relaté dans ce journal $[29,30]$. Ils sont évoqués dans la prévention de la dégénérescence maculaire liée à l'âge [31] et des troubles cognitifs associés au vieillissement [26, 27].

\section{Matériels et méthodes}

Calcul de la consommation par habitant et par an des principales espèces ou principaux groupes d'espèces de poissons et fruits de mer en France. La méthode du bilan d'approvisionnement alimentaire, définie par la FAO [32] a été retenue, avec quelques modifications. Cette méthode permet de calculer un bilan d'approvisionnement pour l'année 2005 à partir de deux ensembles de données statistiques officielles (OFIMER, douanes, ministère des Finances, ministère de I'Agriculture et de la Pêche). La première est la production de produits primaires de la pêche et de l'aquaculture destinés à I'alimentation humaine uniquement, évidemment à l'exclusion de ceux destinés à I'alimentation animale et à un usage industriel non alimentaire. Le deuxième chiffre les importations et les exportations de produits de la pêche et de l'aquaculture destinés à l'alimentation humaine, qu'il s'agisse des produits primaires (bruts, animaux entiers) ou des produits transformés.
Pour les poissons et fruits de mer, la FAO [33] recommande de réaliser les calculs en équivalent poids vif, ce qui exige la connaissance des coefficients de conversion. Ceux-ci sont définis en pratique par le poids de produit vivant nécessaire pour obtenir une unité de poids de produit transformé. C'est ainsi que les données de production (importation et exportation) sont converties en équivalent poids vif; en utilisant ces coefficients de conversion. Ensuite, la consommation par habitant est calculée selon la formule suivante: Consommation par habitant $=($ Production + Importations - Exportations $) /$ Population .

Malheureusement, les coefficients de conversion sont établis à un niveau très agrégé par la FAO (pour certains groupes d'espèces), ils ne permettent pas une connaissance au niveau de l'espèce ou même du groupe d'espèces. Par conséquent, d'autres coefficients de conversion à un niveau plus désagrégé ont été établis [34]. En comparant la consommation de huit pays européens par grandes familles de poissons et fruits de mer, une approche encore plus fine a été réalisée pour la France [35]. Par ailleurs, des coefficients ont été établis pour les principales espèces consommées en France à partir des documents édités par les différents centres techniques spécialisés en poissons et fruits de mer, ainsi qu'à partir d'enquêtes auprès des opérateurs de la filière. À titre d'illustration, le coefficient de conversion d'un filet de saumon est de 1,68 tandis que celui d'un filet de cabillaud est de 2,63.

Pour les données de production et surtout par celles du commerce extérieur, il existe des variations interannuelles, elles sont fréquemment la conséquence de décalages temporels dans les déclarations émanant des entreprises. Ces biais statistiques sont écartés en calculant la consommation par habitant sur la moyenne des trois dernières années. Ce calcul, sur la moyenne des trois dernières années, évalue la consommation totale de poissons et fruits de mer en France à 34,7 kg par habitant et par an, en équivalent poids vif, ce qui est considéré comme reflétant la consommation en 2005. Ce travail fournit donc la quantité de poissons et fruits de mer (entiers) consommés par an et par habitant en France, qu'ils soient issus de la production nationale ou importés, et quel que soit le niveau de transformation sous lequel ils apparaissent dans les données statistiques (produits entiers ou découpés, frais, surgelés ou en conserve, fumés, etc.).

Le calcul suivant est celui de la quantité de chair ingérée par les Français à partir de ces poissons et fruits de mer, en utilisant de nouveau et en sens inverse (si besoin) les coefficients de conversion entre poids vif et poids de filet de poisson ou de fruit de mer décortiqué. À partir de la consommation apparente par habitant exprimée en $\mathrm{g} / \mathrm{j}$, les portions réellement absorbées ont donc été déterminées d'après les tables françaises [36], et à partir de données et calculs personnels. Les estimations des portions comestibles et absorbées (c'est-à-dire supposées intégralement consommées) sont hautes, car le filetage industriel induit de plus grandes pertes, et la consommation domestique néglige parfois une fraction importante du poisson.

Pour ce qui concerne les teneurs en nutriments, les calculs ont été réalisés à partir des tables de compositions [36-40], et le site Internet USDA « database, nutrient data laboratory ». De nombreuses publications (au nombre de 43) ont été examinées, parmi lesquelles : [15, 16, 41-50]. Les valeurs de références présentées dans le tableau sont des moyennes pondérées. Certains chiffres, très écartés des valeurs habituelles, ont été exclus dans les calculs. Certaines espèces, non consommées en France, ont été exclues (consulter la légende du tableau).

Par exemple, le Saumon du Pacifique n'est pas pris en compte, car cette espèce non pas Salmo, mais Oncorhynchus, est pratiquement absente de la consommation française ; elle contient beaucoup moins d'acides gras oméga-3. Le saumon atlantique de l'hémisphère sud, tel celui de Tasmanie [51] n'a pas non plus été pris en compte (EPA + DHA : 1,93mg/100 de filet sans la peau), car il n'est pas importé en France. Pour certaines espèces, aucune valeur publiée est susceptible d'être considérée comme 
Tableau 1

\begin{tabular}{|ll|}
\hline $\begin{array}{l}\text { Les } 10 \text { poissons et fruits de mer les plus consommés en } 2005 \\
\text { (total chair consommable achetée) }\end{array}$ \\
\hline g/jour/habitant & \\
\hline Thon & 4,8 \\
Cabillaud & 4,0 \\
Saumon & 3,8 \\
Lieu d'Alaska & 3,3 \\
Sardine & 2,0 \\
Lieu noir & 1,7 \\
Crevette & 1,7 \\
Moule & 1,4 \\
Merlu & 1,4 \\
Hareng & 1,3 \\
Maquereau & 1,2 \\
Baudroie & 1,1 \\
Total chair consommable achetée & 36,4 \\
g/jour/habitant & \\
\hline
\end{tabular}

fiable : pour les oursins par exemple. Cela ne modifie pas les conclusions de ce travail, car leur consommation est très faible. Pour quelques espèces d'importation récente, comme le tilapia, les rares données sont le fruit de travaux réalisés en Afrique ou en Afrique du Sud [52] affichent des amplitudes considérables dans les teneurs en nutriments selon les variétés : pour le tilapia du Mozambique (Oreochromis mossambicus) le DHA est de 3,3\% des acides gras totaux, pour le tilapia du Nil (Oreochromis niloticus) il est de 3,8\%, pour le « banded tilapia (Tilapia sparrmanii) il est de 3,8\% et pour le tilapia rouge (Oreochromis niloticus) il est de $8,5 \%$. La quantité absorbée reste donc faible, d'une part parce que la part du DHA parmi les acides gras est modeste et d'autre part car s'agit de poissons maigres.

Dans le tableau 2, les chiffres présentés pour les teneurs en DHA de chaque espèce, ne sont que des moyennes. En effet, il y a des variations selon les auteurs, les lieux géographiques de pêche ou de culture, d'autant que le niveau anatomique sur lequel les dosages ont été effectués n'est fréquemment pas précisé. Or les différences de teneurs en lipides peuvent être considérables. Dans un même filet de saumon, par exemple, elles varient selon la localisation de $2,4 \%$ à près de $18,6 \%$ [53]. D'où l'intérêt de préciser soigneusement la région anatomique sur laquelle les dosages ont été réalisés [45], ce qui n'est malheureusement pas fréquemment le cas.

Le thon, poisson le plus consommé en France, se révèle un très gros contributeur dans la couverture de DHA. En France, il est relativement peu acheté frais par les ménages, mais il est présent soit en conserve (notamment appertisée), soit en restauration. Le thon en conserve est constitué pour $70 \%$ d'albacore, pour $28 \%$ de listao et pour $2 \%$ de germon.

Les conclusions sont basées sur les teneurs en nutriments des produits crus.

\section{Résultats et discussion}

À titre d'indication, les dix poissons les plus consommés sont identifiés dans le tableau 1. Les calculs ne sont pas faits sur le poids de produit frais entier, mais sur la partie consommable, donc théoriquement consommée.

Les 10 poissons et fruits de mer (tableau 2) les plus riches en DHA sont donc : le maquereau (1 $490 \mathrm{mg} / 100 \mathrm{~g}$ de portion), la sardine (1 350), le saumon (1 247), le hareng (937), les squales (660), l'anchois (600), la truite (540), le calmar (490), le bar (430), et l'anguille et le thon (430).
Les plus forts contributeurs à la couverture en DHA dans I'alimentation actuelle, base de la présentation du tableau 2, sont le saumon, la sardine, le thon, le maquereau, le hareng, le lieu d'Alaska (colin), le cabillaud (morue), la truite, le merlu, le lieu noir.

Dans le tableau 2, les 34 espèces de poissons et de fruits de mer (pour lesquelles les teneurs en DHA sont connues) représentent $150 \%$ des ANC. La prise en compte de la totalité des espèces individualisées (en extrapolant à celles dont les consommations ou les teneurs en DHA ne sont pas connues) donne $156 \%$ des ANC. Les 5 poissons parmi les plus gras (saumon, sardine, thon, maquereau et hareng) participent pour $63 \%$ parmi les 34 espèces dont les teneurs en DHA sont connues, ce qui représente tout de même $95 \%$ des ANC.

Ainsi donc, comme montré dans le tableau 2, la consommation de DHA serait satisfaite en France par le seul apport des poissons et de fruits de mer, puisqu'ils couvrent $156 \%$ des ANC, ce qui est d'ailleurs en accord [1] avec l'étude SU.VI.MAX [54]. Toutefois, cette observation doit être modérée et pondérée par diverses considérations.

\section{Les ANC français pour le DHA sont bas}

Les ANC français, tels que définis en 2000 [4], sont plus faibles que ceux proposées dans d'autres pays ou par d'autres instances. C'est ainsi que I'ISFFAL propose $220 \mathrm{mg}$ de DHA au minimum.

\section{Distinction de l'effet pharmacologique et l'apport nutritionnel}

En effet, les quantités proposées pour les ANC ont pour objectif d'assurer une bonne couverture nutritionnelle. Or, les acides gras oméga-3, notamment I'EPA et le DHA, présentent des effets pharmacologiques, toutefois à des doses nettement supérieures. Ceci est bien connu dans le domaine cardio-vasculaire. Les doses médicamenteuses efficaces sont donc nettement supérieures aux ANC : elles se situent globalement vers $1000 \mathrm{mg} \mathrm{d}^{\prime} \mathrm{EPA}+\mathrm{DHA}$. Dans ce sens, la consommation d'EPA+DHA, dans les poissons et les fruits de mer, est insuffisante en France. D'autre part, les femmes enceintes ou allaitantes ont des besoins très accrus (multipliés par 2,5 pour ce qui concerne le DHA), la consommation actuelle de poissons et fruits de mer est donc insuffisante pour elles, y compris dans le strict cadre des ANC.

Si l'on compare les doses nutritionnelles et pharmacologiques, il est intéressant de noter que l'homme qui absorbe le plus de DHA dans la cohorte SU.VI.MAX (soit $1472 \mathrm{mg} /$ jour) [1] en consomme tout de même moins que l'esquimau «moyen » du même âge actuellement (1 596 $\mathrm{mg} /$ jour) [55]. De plus, lui-même en absorbe moins que ses parents, sur lesquels les observations épidémiologiques ont effectivement été portées. Dans la condition la plus favorable, en particulier au niveau cardiovasculaire, la consommation de poissons et de fruits de mer en France est manifestement très insuffisante pour exercer un effet pharmacologique.

\section{Cohérence avec d'autres données}

D'après SU.VI.MAX, les adultes français [1] consomment (dans l'ensemble de leurs aliments (poissons et fruits de mer inclus) en moyenne 273 $\mathrm{mg} /$ jour de DHA pour les hommes et $226 \mathrm{mg} /$ jour pour les femmes (moyenne de $249 \mathrm{mg} / \mathrm{jour}$ ), ce qui représente donc environ 2 fois plus que les ANC français. Cependant, ces moyennes occultent des disparités considérables selon les individus, allant de moins de 1/12 des ANC à au-delà de 12 fois les ANC. Plus précisément, la consommation minimale est de $10 \mathrm{mg} /$ jour pour les hommes et de $6,5 \mathrm{mg} /$ jour pour les femmes, alors que les maximums sont de $1472 \mathrm{mg} /$ jour pour les hommes et de $1770 \mathrm{mg} /$ jour pour les femmes. En fait, le $5^{\mathrm{e}}$ percentile est de $66 \mathrm{mg} /$ jour pour les hommes et de $50 \mathrm{mg} /$ jour pour les femmes, le $95^{\mathrm{e}}$ percentile étant de $668 \mathrm{mg} /$ jour pour les hommes et de $574 \mathrm{mg} /$ jour pour les femmes. Il est possible que la cohorte SU.VI.MAX ne soit pas parfaitement représentative, car sélectionnée par son implication dans l'alimentation, 
Tableau 2. Poissons et fruits de mer : consommation en France en 2005, teneurs en DHA, et contribution aux ANC du DHA. Les deux premières colonnes représentent le poids frais des animaux entiers, c'est-à-dire : têtes, viscères, squelette, coquilles, etc. La troisième ne prend en compte que la partie comestible de l'animal, vendue, donc supposée absorbée par les consommateurs. Pour éviter toute confusion, les noms des produits sont donnés en anglais (entre parenthèses). Les lignes « autres poissons marins filetés» et «autres poissons marins non filetés ", sont constituées de diverses espèces mal déclarées aux services du Ministère des Finances ou des Douanes, ou non différentiées (sabre, rouget-barbet, raie, turbot, mulet). Le brochet et la perche ne sont pas individualisés. Les données de consommation du sprat, du chinchard et de quelques poissons pélagiques (autres que ceux mentionnés dans ce tableau) sont peu fiables. II n'est donc pas possible de conclure pour eux, si ce n'est que leur contribution est certainement marginale, en France tout au moins. Leur omission ne modifie donc pas l'approximation du résultat total. Ces inconnues représentent $16 \%$ de la consommation en poids, qui ne peut pas être prise en compte dans les évaluations. Seul le saumon atlantique est pris en compte, le tilapia ne l'est pas, faute de données. Seules les valeurs concernant les saumons d'élevage et des truites d'élevage sont prises en compte, car ces espèces ne sont pratiquement pas consommées sauvages en France Les ANC retenus ne sont que ceux d'un homme adulte, car les consommations des poissons et des fruits de mer, selon les sexes et les âges de la vie, ne sont pas connues. La ligne « total » représente le total calculé pour le total des espèces individualisées, soit 73,58 g/jour/habitant en poids frais, sur un total général de 95,53 g/jour/habitant ; elle ne prend pas en compte les 6 lignes du tableau dénommées " autres ", ce qui représente $21 \%$ de la consommation en poids frais. L'extrapolation aux espèces individualisées dont les teneurs en tel ou tel paramètre n'est pas connue se base sur un recalcul qui repose sur la consommation en poids frais (deuxième colonne). Ainsi, ces espèces individualisées, mais dont les teneurs en tel ou tel paramètre n'est pas connue, représentent (en poids frais) 5,4\% pour le DHA. Ce dernier chiffre a donc permis de calculer les «\%ANC recalculé » est basé sur le total des espèces.

\begin{tabular}{|c|c|c|c|c|c|}
\hline & $\begin{array}{c}\text { Total } \\
\text { kg/an/hab. }\end{array}$ & $\begin{array}{c}\text { Total } \\
\text { g/jour/hab. }\end{array}$ & $\begin{array}{c}\text { Consommable } \\
\text { g/jour/hab. }\end{array}$ & $\begin{array}{c}\text { DHA } \\
\mathrm{mg} / 100 \mathrm{~g} \\
\text { comestible }\end{array}$ & $\begin{array}{c}\text { DHA } \\
\mathrm{mg} / \text { jour } / \mathrm{hab}\end{array}$ \\
\hline Saumon (salmon) & 1,95 & 5,4 & 3,9 & 1247 & 48,0 \\
\hline Sardine & 1,05 & 2,9 & 2,0 & 1350 & 27,3 \\
\hline Thon (tuna) & 3,90 & 10,7 & 4,8 & 420 & 20,2 \\
\hline Maquereau (maquerel) & 0,61 & 1,7 & 1,2 & 1493 & 17,5 \\
\hline Harreng (herring) & 0,82 & 2,3 & 1,4 & 937 & 12,6 \\
\hline Lieu d'Alaska, colin (Alaskan pollock) & 1,84 & 5,0 & 3,3 & 259 & 8,5 \\
\hline Cabillaud, morue (cod) & 1,74 & 4,8 & 4,1 & 170 & 6,9 \\
\hline Truite (trout) & 0,64 & 1,8 & 1,1 & 543 & 5,7 \\
\hline Merlu (hake) & 0,87 & 2,4 & 1,4 & 338 & 4,7 \\
\hline Lieu noir (saithe) & 0,98 & 2,7 & 1,8 & 259 & 4,5 \\
\hline Moules (mussel) & 2,60 & 7,1 & 1,4 & 178 & 3,5 \\
\hline Anchois (anchovy) & 0,29 & 0,8 & 0,6 & 600 & 3,5 \\
\hline Calmar (squid) & 0,29 & 0,8 & 0,7 & 491 & 3,5 \\
\hline Crevette (shrimp) & 1,60 & 4,4 & 1,7 & 160 & 2,8 \\
\hline Squales (sharks) & 0,23 & 0,6 & 0,4 & 660 & 2,6 \\
\hline Coquille St Jacques (scallop) & 2,01 & 5,5 & 1,0 & 144 & 1,4 \\
\hline Baudroie (monk) & 0,48 & 1,3 & 1,1 & 126 & 1,4 \\
\hline Bar (seabass) & 0,13 & 0,4 & 0,2 & 434 & 1,0 \\
\hline Huitres (oysters) & 1,81 & 5,0 & 0,6 & 107 & 0,6 \\
\hline Merlan (whitting) & 0,31 & 0,9 & 0,5 & 107 & 0,6 \\
\hline Lieu jaune (pollack) & 0,11 & 0,3 & 0,2 & 259 & 0,5 \\
\hline Egelfin (haddock) & 0,18 & 0,5 & 0,3 & 139 & 0,5 \\
\hline Flétan (hallibut) & 0,06 & 0,2 & 0,1 & 318 & 0,4 \\
\hline Sole & 0,16 & 0,4 & 0,3 & 141 & 0,4 \\
\hline Sébaste (redfish) & 0,25 & 0,7 & 0,5 & 70 & 0,4 \\
\hline Plie, carrelet (plaice) & 0,07 & 0,2 & 0,2 & 193 & 0,3 \\
\hline Crabe (crab) & 0,44 & 1,2 & 0,2 & 113 & 0,3 \\
\hline Seiche (cuttlefish) & 0,12 & 0,3 & 0,3 & 66 & 0,2 \\
\hline Carpe (carp) & 0,08 & 0,2 & 0,1 & 108 & 0,1 \\
\hline Homard (lobster) & 0,11 & 0,3 & 0,1 & 142 & 0,1 \\
\hline Poulpe (octopus) & 0,05 & 0,1 & 0,1 & 81 & 0,1 \\
\hline Langouste (spiny lobster) & 0,08 & 0,2 & 0,1 & 80 & 0,1 \\
\hline anguille (eel) & 0,00 & 0,0 & 0,0 & 430 & 0,0 \\
\hline Ecrevisse (crayfish) & 0,02 & 0,1 & 0,0 & 12 & 0,0 \\
\hline Langoustine (nephrops) & 0,30 & 0,8 & 0,3 & & \\
\hline Sprat + chinchard + autres & 0,40 & 1,1 & & & \\
\hline Dorade (seabream) & 0,11 & 0,3 & 0,2 & & \\
\hline Lingue (ling) & 0,17 & 0,5 & 0,3 & & \\
\hline Foies, œufs, laitances (roe and lever) & 0,03 & 0,1 & 0,1 & & \\
\hline Autres poissons plats & 0,27 & 0,7 & & & \\
\hline Autres poissons marins (other whole groundfish) & 1,78 & 4,9 & & & \\
\hline Autres poissons marins filetés (other whole goundfish in fillet) & 3,97 & 10,9 & & & \\
\hline Autres poissons eau douce & 0,72 & 2,0 & & & \\
\hline
\end{tabular}




\begin{tabular}{|c|c|c|c|c|c|}
\hline & $\begin{array}{c}\text { Total } \\
\mathrm{kg} / \mathrm{an} / \mathrm{hab} .\end{array}$ & $\begin{array}{c}\text { Total } \\
\text { g/jour/hab. }\end{array}$ & $\begin{array}{c}\text { Consommable } \\
\text { g/jour/hab. }\end{array}$ & $\begin{array}{c}\text { DHA } \\
\mathrm{mg} / 100 \mathrm{~g} \\
\text { comestible }\end{array}$ & $\begin{array}{c}\text { DHA } \\
\mathrm{mg} / \text { jour } / \mathrm{hab}\end{array}$ \\
\hline Autres mollusques et invertébrés & 1,22 & 3,3 & & & \\
\hline Autres crustacés & 0,03 & 0,1 & & & \\
\hline Total & 34,88 & 95,5 & 36,4 & & 180,0 \\
\hline Total espèces individualisées et dosées & & 73,6 & & & \\
\hline \%ANC du DHA & & & & & 150 \\
\hline Total recalculé sur toutes les espèces & & & & & 187 \\
\hline$\%$ ANC total recalculé & & & & & 156 \\
\hline
\end{tabular}

et correspondant à des tranches d'âge particulières (35-63 ans pour les femmes, 45-63 ans pour les hommes). D'autres variations, selon les personnes, peuvent être la conséquence de localisations géographiques : ainsi, la seule région ouest de la France assure $30 \%$ de la consommation nationale [35]. Enfin, les ANC sont aisément atteints en consommant 1 fois par semaine une portion de l'un des poissons gras (sardine, maquereau, saumon et hareng), ce qui est effectué par les individus dont la ration de protéines est majoritairement d'origine aquatique.

Dans l'étude SU.VI.MAX [1], les poissons contribuent pour $65 \%$ de l'apport en DHA, à raison de $51 \%$ pour ceux qui sont gras, $23 \%$ pour les maigres ; les fruits de mer participent pour $21 \%$ (bien que peu gras, ils sont largement consommés), ce qui est en accord avec le fait que le quatrième contributeur en apport alimentaire en DHA est le lieu d'Alaska, comme le montre le tableau 2. Même les fruits de mer contribuent finalement significativement aux apports. De ce fait, d'après SU.VI.MAX, la contribution des poissons et fruits de mer est de $162 \mathrm{mg} /$ jour du même ordre de grandeur que le chiffre présenté dans le tableau de ce travail (180 mg), quoique calculé de manière radicalement différente.

II convient donc de cibler les petits consommateurs de DHA, pour les convaincre d'absorber beaucoup plus de poissons et fruits de mer.

Incidemment, l'intérêt fondamental et spécifique des poissons et fruits de mer dans I'approvisionnement alimentaire en DHA est montré sur un effectif mixte (relativement faible), situé dans la région de Bretagne, à qui il était demandé de ne pas manger de poissons ni de fruits de mer. Sa consommation était inférieure à la moitié des ANC [2], de $70 \mathrm{mg}$ pour le DHA ; passant à $140 \mathrm{mg}$ par l'absorption d'aliments issus d'animaux nourris avec des graines de lin [communication personnelle].

En pratique, la consommation de capsules d'huiles de chair de poisson, et encore plus de leurs succédanés, ne se justifie pas en termes de compléments alimentaires sans examen des habitudes alimentaires individuelles permettant de détecter les petits consommateurs de produits de la pêche et de l'aquaculture. En revanche, ces capsules sont d'indication médicale, mais à des doses qui dépassent largement les ANC pour ce qui est des quantités de DHA (I'EPA ne bénéficiant pas d'ANC) en prévention d'accident ischémique cardio-vasculaire, après une alerte ou un premier incident; i et bien évidemment à la suite d'un accident ischémique patent. Dans une certaine mesure, il est possible d'envisager de remplacer ces capsules par la consommation d'au moins une part quotidienne de poisson gras.

\section{Incidences des méthodes de préparation culinaires ou de conservations}

Elles ne sont pas prises en compte dans ce travail, car elles sont largement inconnues, mais ne sont certainement pas négligeables, voire importantes dans le cas des fritures profondes; elles sont très différentes selon les cas et les espèces. De plus, l'appertisation, et, dans une plus grande mesure la friture, font migrer une fraction notable des acides gras oméga-3 dans une huile qui n'est que très partiellement consommée. Tous les chiffres utilisés sont basés sur les produits crus, mais achetés en fait par le consommateur sous quelques formes que ce soit (frais, congelés, appertisé ou sous toute autre forme de conserve, dans les plats préparés, mangés chez soi, en restauration collective, etc.). Or, tout n'est pas consommé.

\section{Rôle prépondérant d'une seule espèce, qui peut être remis en question}

Le saumon contribue pour environ $27 \%$ aux apports en DHA, mais ce chiffre est obtenu sur la base de la composition du saumon d'élevage nourri avec des huiles de poisson [45]. Ce qui représente $92 \%$ de la consommation actuelle de saumon en France. Cette contribution à la couverture en DHA pourrait s'avérer beaucoup plus faible si les saumons proposés sur le marché étaient moins gras (le saumon d'élevage est actuellement presque 2 fois plus gras que celui qui est sauvage [45]) ou nourris avec des corps gras pauvres ou dépourvus en acides gras oméga-3.

\section{Conclusion}

Dans les calculs présentés dans le tableau 2, il est présumé que toutes les portions comestibles sont consommées, ce qui n'est pas réalisé en pratique. Par ailleurs, les pertes lors des préparations culinaires et des techniques de conservation (notamment appertisés), très variables et fort mal connues, ne sont pas prises en compte, car peu mesurées. Les chiffres sont donc alors surévalués. En revanche, $16 \%$ des poissons et fruits de mer consommés ne sont pas pris en compte, car non pris recensés dans les statistiques officielles, du fait de leur faible consommation générale, ou de leur utilisation très locale. Les chiffres présentés dans le tableau 2 sont donc par conséquent sous-évalués. II est possible que la sur- et la sous-évaluation se compensent. Les conclusions restent donc manifestement valables.

Pour ce qui concerne les acides gras oméga-3, seuls I'EPA et le DHA ont été retenus. II convient toutefois de souligner que les teneurs en ALA sont notables dans les poissons et les fruits de mer, voire très importantes en aquaculture si les poissons sont nourris avec des produits issus du colza ou du lin [56-58]. Car, pour les poissons comme pour les autres espèces, la qualité des aliments qui leurs sont donnés influent sur la valeur nutritionnelle pour l'homme qui les consomme; en effet, pour les poissons, il existe une relation étroite entre les lipides alimentaires (taux et nature) et les lipides corporels de la fraction comestible $[5,7,59]$. Par ailleurs, outre les acides gras oméga-3, les poissons et les fruits de mer contiennent des quantités notables d'acides gras mono-insaturés, potentiellement intéressants, généralement de la famille oméga-9 (celle de l'acide oléique), dont l'acide nervonique. Or, le caractère semiindispensable de l'acide oléique a été proposé $[60,61]$.

En plus du DHA, il convient de rappeler que nombre de produits de la pêche et de l'aquaculture contiennent aussi de notables quantités de plusieurs vitamines, en particulier D et B12 (mais aussi, mais dans une moindre mesure, la vitamine $A$ et les vitamines du groupe $B$, notamment 
PP), de minéraux et d'éléments traces, au premier chef l'iode et le sélénium (mais aussi magnésium, fer, et même de faibles quantités de fluor). Les teneurs en vitamine $\mathrm{E}$ des poissons et des fruits de mer sont généralement faibles, mais elles peuvent être accrues dans les produits de l'aquaculture.

Le seul aliment qui apporte de quantités notables de DHA (et d'ALA) est l'œuf "sauvage ", ou bien celui issu de poules nourries de manière pertinente [10]. Les algues pourraient constituer une voie d'avenir [62, 63]. La discussion portant sur la biodisponibilité des acides gras oméga-3 selon la position sur le triglycéride sort du cadre de ce travail, il est à noter que les phospholipides sont d'intérêt particulier [64], car la biodisponibilité de leurs acides gras est intéressante [65]. Globalement, les biodisponibilités des acides gras oméga- 3 des poissons sont meilleures dans le poisson que dans des capsules contenant des esters éthyliques : 9 fois supérieures pour le DHA [66, 67]. Les perspectives d'utilisation des huiles marines sont prometteuses, grâce aux techniques d'extraction, de fractionnement et de concentration $[68,69]$.

L'intérêt de l'élevage (l'aquaculture), outre le fait qu'il évite le dépeuplement des mers, et d'assurer une teneur normalisée en lipides et en acides gras oméga-3. L'avantage de l'élevage est d'assurer une composition constante, indépendante des variations observées chez l'animal sauvage, par exemple saisonnières, géographiques ou sexuelles. L'élevage constitue une alternative incontournable pour l'alimentation humaine, notamment pour ce qui concerne le saumon [45].

L'AFSSA a émis [70] un avis quant aux allégations possibles, compte tenu des connaissances scientifiques et médicales, en se restreignant toutefois aux seules maladies cardio-vasculaires. Quoi qu'il en soit, un aliment est « riche en acides gras oméga- 3 » quand il fournit au moins $30 \%$ des ANC en acide alphalinolénique (ALA) ou en DHA par jour pour : soit $100 \mathrm{~g}$ d'aliment, soit 100 millilitres, soit 100 kilocalories. Pour les hommes, cela représente quotidiennement soit $0,6 \mathrm{~g}$ d'ALA soit $0,036 \mathrm{~g}$ de DHA. L'EPA n'est pas pris en considération. Pour ce qui est du DHA, presque tous les poissons, produits de l'aquaculture et fruits de mer répondent actuellement à cette définition. D'autant que les teneurs en cholestérol et en acides gras saturés des poissons et des fruits de mer sont généralement modestes.

Négliger les poissons et les fruits de mer peut donc engendrer à des déséquilibres alimentaires préjudiciables à la santé, du fait de leur richesse en nombre de nutriments, dont le DHA. Finalement, parmi l'ensemble des aliments, nombre de poissons et de fruits de mer fournissent le DHA le moins onéreux (en particulier les conserves appertisées de poisson).

Remerciements. Ce travail a été financé par l'Ofimer et I'Inserm.

\section{RÉFÉRENCES}

1. ASTORG P, ARNAULT N, CZERNICHOW S, NOISETTE N, GALAN P, HERCBERG S. Dietary intakes and food sources of n-6 and n-3 PUFA in French adult men and women. Lipids $2004 ; 39: 527-35$.

2. WEILL $P$, SCHMITT B, CHESNEAU G, DANIEL $N$, SAFRAOU F, LEGRAND P Effects of introducing linseed in livestock diet on blood fatty acid composition of consumers of animal products. Ann Nutr Metab 2002 ; 46 : 182-91.

3. COMBE N, BOUE C. Apports alimentaires en acides linoléique et alphalinolénique d'une population d'Aquitaine. OCL $2001 ; 8: 118-21$.

4. LEGRAND P, BOURRE JM, DESCAMPS B, DURAND G, RENAUD S. In : Lipides. Apports nutritionnels conseillés pour la population française. Martin A. Tec et Doc Lavoisier, $2000: 63-82$.

5. BOURRE JM. Alimentation animale et valeur nutritionnelle induite sur les produits dérivés consommés par l'homme : les lipides sont-ils principalement concernés? OCL $2003 ; 10$ : 405-24.

6. COMBE N, FENARTE. Les oméga-3: de I'alimentation à la nutrition humaine. OCL $2004 ; 11: 46-9$.
7. BOURRE JM. Where to find omega-3 fatty acids and how feeding animals with diet enriched in omega-3 fatty acids to increase nutritional value of derived products for human: what is actually useful? I Nutr Health Aging $2005 ; 9: 232-42$

8. COMBE N, BOUÉ-VAYSSE C. Face aux besoins et à la réalité des consommations, quelles sont les spécificités des différentes sources d'acides gras oméga-3 disponibles. OCL 2004 ; 11 : 103-5.

9. MOUROT J, HERMIER D. Lipids in monogastric animal meat. Reprod Nutr Dev $2001 ; 41: 109-18$.

10. BOURRE JM. L'œuf naturel multi-enrichi : des apports élevés en nutriments, notamment acides gras oméga-3, en vitamines, minéraux et caroténoïdes. Méd Nutr $2005 ; 41$ : 116-34.

11. MEDALE F, LEFEVRE F, CORRAZE G. Qualité nutritionnelle et diététique des poissons : constituants de la chair et facteurs de variation. Cah Nutr Diet $2003 ; 1: 37-44$.

12. MARIOJOULS M. Perception de la qualité des produits de la pêche et de I'aquaculture par le consommateur. Cah Nutr Diet 2003 ; 38 : 29-36.

13. FAUCONNEAU B. La qualité nutritionnelle des poissons d'élevage, un concept multifactoriel polymorphe. Cah Nutr Diet 2003 ; 38 : 53-7.

14. RAZANMAHEFA L, LAFAY L, OSEREDCZUK M, et al. Consommation lipidique de la population française et qualité des données de composition des principaux groupes d'aliments vecteurs. OCL $2005 ; 92$ : 647-57.

15. BOURRE JM. Impact de l'enrichissement avec les acides gras oméga-3 de I'alimentation des animaux sur leurs produits consommés par l'homme. Med Sci (Paris) $2005 ; 21: 773-9$

16. BLANCHET C, LUCAS M, JULIEN P, MORIN R, GINGRAS S, DEWAILLY E. Fatty acid composition of wild and farmed Atlantic salmon (Salmo salar) and rainbow trout (Oncorhynchus mykiss). Lipids 2005 ; 40 : 529-31.

17. HARRIS WS. Are omega- 3 fatty acids the most important nutritional modulators of coronary heart disease risk? Curr Atheroscler Rep 2004 ; 6 : 447-52.

18. LECERF JM. Poisson, acides gras oméga-3 et risque cardiovasculaire : données épidémiologiques. Cah Nutr Diet 2004 ; 39 : 143-50.

19. DUCIMETIERE P. Acides gras omega- 3 et maladies cardio-vasculaires: approche épidémiologique. OCL $2004 ; 11: 66-9$.

20. HE K, SONG Y, DAVIGLUS M, et al. Accumulated evidence on fish consumption and coronary heart disease mortality. A meta-ananlysis of cohort studies. Circulation $2004 ; 109: 2705-11$.

21. MOZAFFARIAN D, GOTTDIENER JS, SISCOVICK DS. Intake of tuna or other broiled or baked fish versus fried fish and cardiac structure, function, and hemodynamics. Am / Cardiol 2006 ; 97 : 216-22.

22. CHAJES V, BOUGNOUX P. Omega-6/omega-3 polyunsaturated fatty acid ratio and cancer. World Rev Nutr Diet $2003 ; 92$ : 133-51.

23. NORAT T, BINGHAM S, FERRARI $P$, et al. Meat, fish, and colorectal cancer risk : the European Prospective Investigation into cancer and nutrition. / Natl Cancer Inst 2005 ; 97 : 906-16.

24. ASTORG P. Acides gras alimentaires, cancer colo-rectal et cancer de la prostate : études épidémiologiques. OCL 2005 ; 92 : 670-94.

25. HEBUTERNE X. Quelle place pour les huiles de poisson au cours des maladies inflammatoires chroniques de l'intestin? OCL 1998 ; 5 : 173-7.

26. BOURRE JM. Effets des nutriments (des aliments) sur les structures et les fonctions du cerveau : le point sur la diététique du cerveau. Rev Neurol $2004 ; 160$ : 767-92.

27. BOURRE JM. Relations entre acides gras oméga-3, oméga-9, structures et fonctions du cerveau. Le point sur les dernières données. Le coût financier alimentaire des oméga-3. OCL $2003 ; 10$ : 165-74.

28. VANCASSEL S. Omega-3 et neurotransmission cérébrale. OCL $2004 ; 11$ : $58-65$ 
29. BOURRE JM. Acides gras oméga-3 alimentaires et neuropsychiatrie. OCL $2004 ; 11: 362-70$.

30. BOURRE JM. Dietary omega- 3 fatty acids and psychiatry : mood, behaviour stress, depression, dementia and aging. / Nutr Health Aging 2005 ; 9 : 31-8.

31. DESMETTRE T, LECERF JM, SOUIED EH. Nutrition and age-related macular degeneration. J Fr Ophtalmol $2004 ; 27$ : 3S38-3556.

32. FAO. Bilans alimentaires, moyenne 1998-2000. Rome, Italia : Food and agriculture organization of the United Nations, 2002.

33. FAO. Fish and fishery products - World apparent consumption statistics based on food balance sheets, 1996-2001. Rome, Italia: Food and agriculture organization of the United Nations, 2004.

34. PAQUOTTE P, MARIOJOULS C, YOUNG J. Seafood market studies for the introduction of new aquaculture products. Cahiers Options Méditerranéennes 2002 ; vol. 59.

35. GIRARD S, PAQUOTTE P. La consommation de produits de la pêche et de I'aquaculture en France. Cah Nutr Diet $2003 ; 1$ : 17-28.

36. FAVIER JC, IRELAND-RIPERT J, TOQUE C, FEINBERG M. Répertoire général des aliments, table de composition. INRA, CNERNA-CIQUAL, Tec et Doc Lavoisier, 1995.

37. LAMAND M, TRESSOL JC, IRELAND-RIPERT J, FAVIER JC, FEINBERG M Répertoire général des aliments, tome 4. Table de composition minérale. Tec et Doc Lavoisier, 1996.

38. SOUCI S, FACHMANN W, KRAUT H. Food composition and nutrition tables. Med Pharm Scientific Publisher Stuttgart. CRC press, 2000.

39. MCCANCE R, WIDDOWSON'S E. The composition of foods. Sixth summary edition. Cambridge : Food Standards Agency. , 2004.

40. SU. VI. MAX. Table de composition des aliments. Paris : Ed. Economica, 2006.

41. ACKMAN RG. Nutritional composition of fats in seafoods. Prog Food Nutr Sci $1989 ; 13: 161-289$.

42. AHLGREN G, BLOMQVIST P, BOBERG M, GUSTAFSSON IB. Fatty acid content of the dorsal muscle-an indicator of fat quality in freshwater fish. J Fish Biol 1994 ; 45 : 131-57.

43. ALASALVAR A, TAYLOR K, ZUBKOV E, SHAHIDI F, ALEXIS M. Differentiation of cultured and wild sea bass (Dicentrarchus labrax) : total lipid content, fatty acid and trace mineral composition. Food Chem 2002 ; 79 : 145-50.

44. BLANCHET C, DEWAILLY E. Le guide alimentaire du Saint Laurent. Ministère de l'agriculture, des pêcheries et de l'alimentation du Québec, 2003.

45. BOURRE JM, OALAND O, BERG LT. Les teneurs en acides gras oméga-3 des saumons Atlantique sauvages ( $d^{\prime} E c o s s e$, Irlande et Norvège) comme références pour ceux d'élevage. Med Nutr $2006 ; 42: 36-49$.

46. CAHU C, SALEN P, DE LORGERIL M. Farmed and wild fish in the prevention of cardiovascular diseases : assessing possible differences in lipid nutritional values. Nutr Metab Cardiovasc Dis 2004 ; 14 : 34-41.

47. KARAKOLTSIDIS P, ZOTOSA, CONSTANTINIDES S. Compositon of commercially important mediterranean finfish, crustaceans, and molluscs. J Food Comp Anal $1995 ; 8$ : 258-73.

48. KING I, CHILDS MT, DORSETT C, OSTRANDER JG, MONSEN ER. Shellfish : proximate composition, minerals, fatty acids, and sterols. I Am Diet Assoc $1990 ; 90: 677-85$

49. FORNERIS G, MORISIO GUIDETTI L, SARRA C. Determinazione quantativa dell'acido eicopentenico in alcune specie di animali aquati. La rivista della societa di scienza dell'alimentazione $1981 ; 10: 155-8$.

50. PUUSTINEN T, PUNNONEN K, UOTILA P. The fatty acid composition of North-European fish species. Acta Med Scand 1985 ; 218 : 59-62.
51. NICHOLS P. Source of long chain omega-3 oils. Lipid technology $2004 ; 16$ : 247-51.

52. CHETTY N, REAVIS SC, IMMELMAN AR, ATKINSON PM, VAN AS JG. Fatty acid composition of some South African fresh-water fish. S Afr Med I 1989; $76: 368-70$.

53. KATITOU P, HUGHES SI, ROBB D. Lipid distribution within Atlantic salmon (Salmo salar) fillets. Aquaculture $2001 ; 202$ : 89-99.

54. HERCBERG S, GALAN P, PREZIOSI P, et al. The SU. VI. MAX Study : a randomized, placebo-controlled trial of the health effects of antioxidant vitamins and minerals. Arch Intern Med $2004 ; 164: 2335-42$.

55. DEWAILLY E, BLANCHET C, LEMIEUX S, et al. n-3 fatty acids and cardiovascular disease risk factors among the inuit of Nunavik. Am / Clin Nutr 2001; 74 : 464-73.

56. REGOST C, ARZEL I, CARDINAL M, ROSENLUND G, KAUSHIK SI. Total replacement of fish oil by soybean or linseed oil with a return to fish oil in turbot (Psetta maxima) : 2 flesh quality roperties. Aquaculture $2003 ; 220$ : 737-47.

57. BELL JG, HENDERSON RJ, TOCHER DR, SARGENT JR. Replacement of dietary fish oil with increasing levels of linseed oil : modification of flesh fatty acid compositions in Atlantic salmon (Salmo salar) using a fish oil finishing diet. Lipids $2004 ; 39$ : 223-32.

58. MENOYO D, LOPEZ-BOTE C], OBACH A, BAUTISTA JM. Effect of dietary fish oil substitution with linseed oil on the performance, tissue fatty acid profile, metabolism, and oxidative stability of Atlantic salmon. J Anim Sci $2005 ; 83$ : 2853-62.

59. CORRAZE G, KAUSHIK S. Les lipides des poisons marins et d'eau douce. OCL $1999 ; 6: 111-5$.

60. BOURRE JM, DUMONT O, DURAND G. Effet-dose de l'acide oléique alimentaire. Cet acide est-il conditionnellement essentiel? OCL 2000 ; 7 : 524-30.

61. BOURRE JM, DUMONT OL, CLEMENT ME, DURAND GA. Endogenous synthesis cannot compensate for absence of dietary oleic acid in rats. I Nutr $1997 ; 127: 488-93$.

62. PENCHREAC'H G, DEVIOS M, POISSON L, HERAULT J, LOISEUAC, ERGAN F. Les micro-algues marines : source alternative d'acide eicosapentaénoïque (EPA et d'acide docosahéxaénoïque (DHA). OCL 2004; 11 : $118-22$.

63. POISSON L, DEVOS M, PENRAC'H G, ERGAN F. Acides gras polyinsaturés des microalgues : intérêt et développement actuels. OCL $2002 ; 9$ : 92-5.

64. FANNI J, LINDER M, PARMENTIER M. Lipides polaires marins. OCL 2004 ; $11: 142-5$.

65. BOURRE JM, DUMONT O. The adminitration of pig brain phospholipids versus soybean phospholipids in the diet during the period of brain development in the rat results in greater increments of brain docosahexaenoic acid. Neurosc Lett $2002 ; 335: 129-33$.

66. VISIOLI F, RISE P, BARASSI MC, MARANGONI F, GALLI C. Dietary intake of fish vs. formulations leads to higher plasma concentrations of $n-3$ fatty acids. Lipids $2003 ; 38: 415-8$.

67. VISIOLIF, RIZE P, MARANGONIF, GALLIC. The intake of long chain omega- 3 fatty acids through fish versus capsules results greater increments of their plasma levels in healthy subjects. OCL $2005 ; 2: 116-7$.

68. LINDER M, FANNI J, PARMENTIER M. Extraction, fractionnement et concentration des huiles marines. OCL $2004 ; 11: 123-30$.

69. TURON F, VILLENEUVE P, PINA M. Amélioration de la qualité nutritionnelle des huiles de poisson. OCL $2004 ; 11: 146-9$.

70. AFSSA. Acides gras de la famille oméga 3 et système cardiovasculaire : intérêt nutritionnel et allégations. Paris : Tec et Doc Lavoisier, 2003. 